\title{
Multi-Agent-based Distributed Optimization for Demand-Side-Management Applications
}

\author{
Tim Dethlefs, Thomas Preisler, Wolfgang Renz \\ Hamburg University of Applied Sciences \\ Faculty of Engineering and Computer Science \\ Berliner Tor 7, 20099 Hamburg, Germany \\ Email: \{tim.dethlefs I thomas.preisler I wolfgang.renz\}@haw-hamburg.de
}

\begin{abstract}
Dynamic and volatile grid conditions caused by the growing amount of renewable energy producers require the operation of large-scale distributed Demand-Side Management (DSM) applications. This is one of the tasks of the aggregator role in smart grid operation according to the Smart Grid Architecture Model (SGAM). For the optimization of distributed demandside loads under such conditions, Multi-Agent Systems (MAS) have been shown to provide an appropriate paradigm to model, simulate and deploy automated operating components.

In this paper, we address an engineering problem that is still a matter of concern, namely the construction of efficient distributed optimization algorithms in conjunction with a generic software architecture. For this purpose, a distributed Multi-Agent architecture is presented with a generic consumer model and an energy exchange market as well as further roles and components. Ant Colony System Optimization is shown to effectively optimize consumers in a nature-inspired, self-organizing way.

The applicability of the proposed approach will be demonstrated in a use-case study where a group of heterogenous consumers optimize their runtimes in order to map their demand to the energy generation of a wind power plant in a self-organized fashion.
\end{abstract}

\section{INTRODUCTION}

$\mathbf{T}$ HE DEVELOPMENT of small, affordable and profitable energy generators as well as the desired growth of renewable energy resources leads to an increasing decentralization and heterogeneity in the smart grid. The capacity and availability of the decentralized energy resources often depends on environmental influences so fast-reacting conventional power plants and energy storages will be needed to compensate such fluctuations.

The utilization of the demand-side potential, the DemandSide-Management (DSM), could be an additional planning option which becomes achievable and affordable through the increasing degree of automation and information- and communication technology (ICT).

The management of both demand and production side can be more efficient and safer with planning and forecasting, but will require predictable and intelligent devices and appliances for domestic households as well as industrial applications. Those devices should be able to coordinate and optimize their schedule in order to achieve pre-qualification for markets or to lower their runtime costs. While in the energy domain no real quality of service, like frequency- or availability services, can be sold, demand-side orientated business models must focus on the flexibility and planning potential of the devices with dynamic pricing or stock exchange models. The scheduling and optimization of the highly heterogeneous and distributed devices needs adaptive solutions. It seems to be likely to use these intelligent embedded devices themselves for this task, because of their computational- and communicationcapacities. So a lightweight and simple optimization algorithm and a distribution concept with a minimum of shared information are required.

Because of the distribution- and autonomy-properties MultiAgent-Systems (MAS) are suitable as a paradigm for the logical representation of grid entities. Even the current topdown modus operandi of the grid could be considered as a distributed MAS-architecture regarding the distribution of loads, substations etc. Therefore, the architectural approach in this paper utilizes the MAS-paradigm towards the distributed optimization of consumers for DSM applications.

The remaining paper is structured as follows: Section II provides an overview on the related literature and covers different aspects of todays grid development towards an emerging DSM integration. In Section III a description of the distributed MAS-Architecture for DSM and optimization is provided. Section IV describes the reference implementation of an adapted Ant Colony System - Algorithm for distributed optimization. In Section V the applicability of the proposed approach is demonstrated in a use-case study where a group of heterogenous consumers optimize their runtimes in order to map their demand to the energy generation of a wind turbine in a self-organized fashion, before Section VI concludes the paper and gives an overview about future work.

\section{RELATED WORK}

Energy supply particularly on the field of electricity currently experiences a profound change. Due to the availability and the versatile ways of use, electricity has become one of the most important energy sources. New applications and potential uses as well as laws, standards, guidelines and social developments generate new requirements for the energy generation, the grid-infrastructure and also the consumer-side. This Section introduces current laws and standards of the energy domain, it gives a brief overview about the situation on the energy markets and presents current grid-infrastructures 
before an overview about related work on DSM and MASSimulation concludes the Section.

\section{A. Laws and Standards}

In the USA the development of energy distribution has mainly arisen from the oil crisis. Until today the National Energy Conservation Policy Act (NECPA) from 1978 regulates the energy consumption in the United States. Contrasting to California, where the electricity-demand has been stabilized by the 1972 decree on device efficiency, building regulation and energy efficiency for energy suppliers, which was manifested as a law in 2006 [1], the power consumption in the rest of the USA has risen steadily [2]. This led to the 2007 Energy Independence and Security ACT (EISA) as an extension of the NECPA. In 2000 Germany applied a law ${ }^{1}$ to regulate the favored input of electricity from renewable energy resources and to guarantee a fixed buyback price for the next 20 years. Until 2020 the amount of electricity generated from renewable resources should have reached at least $35 \%$, rising up to at least $80 \%$ in 2050. In 2013 this amount had already reached $23,4 \%$ [3]. Because of the volatility of renewable energy resources, the favorable input of such unsteady resources conflicts with the classical modus operandi and requires highly dynamic and regulable power plants to compensate energy deficiencies respectively the establishment of corresponding storage capacities as required by the Energy Industry $\mathrm{Act}^{2}$. This law demands that the security and reliability of the energy grid is secured through net- or market-based measures in case of disturbances or compromises. Besides the duties on the producer side, the law also permits the detailed acquisition of performance data and load balancing measurements on the consumer side through load- and time-depended tariffs.

In order to deal with this situation almost all parts of the grid from the producer to the consumer must be automated in the future. This requires a new role- and domain-model as defined by the NIST-Framework-Roadmap [4]. It is established as a quasi-standard for further publications in this area. European organizations and research facilities have adopted the model and extend it for local requirements, e.g. with the extension of the distributed energy resources (DER) domain as part of the European Smart Grid Reference Architecture and the Smart Grid Architecture Model (SGAM), a layered-architecture for smart grids representing the communication and information layers [5].

\section{B. Energy Markets}

Today's electricity market in Europe is highly regulated in order to maintain operational safety. Due to the big trade volume it is mainly accessible for large producers and consumers. The most important energy markets in Europe are the European Energy Exchange $(\mathrm{EEX})^{3}$ respectively the European Power Exchange (EPEX SPOT) ${ }^{4}$ as power-based spot market

\footnotetext{
${ }^{1}$ Gesetz für den Vorrang Erneuerbarer Energien (EEG)

${ }^{2}$ Energiewirtschaftsgesetz (EnWG)

${ }^{3}$ www.eex.com

${ }^{4}$ www.epexspot.com
}

located in Leipzig, Germany. In 2011 the German net-agency (Bundesnetzagentur BNetzA) reformed the bidding conditions for secondary and tertiary control by reducing the minimum submission size, enabling aggregation and allowed deliverance guarantees by third parties. Through the low investment risk and short amortization times both small and medium-sized energy producers as well as DER have gained increasing attractiveness in recent years [6]. But not without creating new challenges: Due to the increasing distribution of energy producers their coordination becomes more and more complex. Also because of their low production rate DER are not able to participate directly at the energy markets. A possible solution is the grouping of DER to larger entities, the so called Virtual Power Plants (VPP) [7], [8].

\section{Communication Infrastructure}

Many projects from the energy domain facilitate internettechnology like the TCP/IP protocol as communication infrastructure for their applications because of the well-defined standards and their wide dissemination [9]. Another interesting infrastructure approach for domestic household automation is given by the OGEMA-Project [10]. It deals with the connection of household devices via a gateway-interface to a coordination centre and allows the automated control of consumers depending on variable power prices. Alongside to the OGEMA-project, OpenADR is an established system for automated demand-response, focusing on top-down business models controlled by a system operator [11]. According to [9] there is still a lack of a common communication standard for demand-side management. [12] points out the need for a registry for DER and consumers which provides information about all available energy services. Therefore, the area-wide facilitation of DSM capacities is still a topic of current research areas.

\section{Demand-Side Management and Multi-Agent Systems}

Demand-Side Management (DSM) is defined by [13] as planning and implementation activities of utilities and operators in order to influence the consumer's demand so that the intended consumer-behavior is achieved. A study of the US Federal Energy Regulatory Commission [14] comes to the conclusion, that in the USA the peak-load will rise from 775 GW in 2009 to $900 \mathrm{GW}$ in 2019. That would require 2000 new power plants. Here DSM is seen as a measurement to limit this growth to $800 \mathrm{GW}$ and to stabilize it in order to deal with the increasing costs and environmental impacts [15]. Apart from energy savings also the purposeful use of volatile effects from the renewable energy resources is a significant DSM use-case in Germany [9].

Multi-Agent Systems (MAS) are an established technology in order to simulate different DSM applications in smart grids. Due to their distribution and autonomy properties they can be developed relatively similar to real infrastructures and grid entities. Almost all approaches share the fact, that agents are used to represent consumers or net-entities. Most approaches utilize the demand-side for feedback control and secondary 


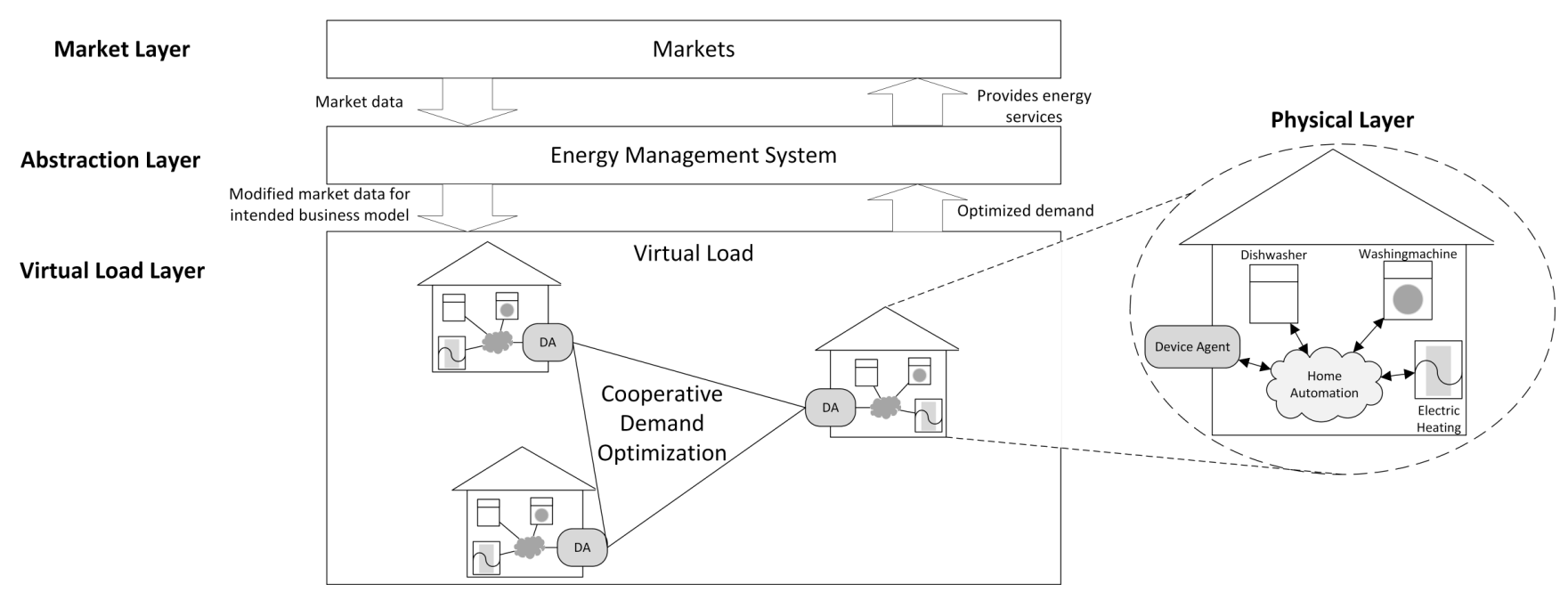

Fig. 1. Architectural model for the distributed optimization system.

and tertiary control energies. Hereby, the examined time frame ranges from near real-time applications as presented in [16] to applications like PowerMatcher [6] which focus more on day ahead planning. The simulation systems mainly differ on how the DSM is achieved. Here it can be differentiated between direct and indirect control. Direct control focuses on the used protocols and signaling, whereas indirect control approaches can be further differentiated between approaches based on price signals, auctions and optimization on the consumerside. An example for the direct control of load is presented in [17] where the joint control of loads is researched. The advantage of this approach is the reliable system response for defined signals, as the connected consumers have to act accordingly to specified signaling. Additionally, the according service provider or operator has complete control over the loads. But this mode of operation implicates hard constraints on the consumer-side concerning the conditions under which load can be switched centrally. Thereby, the coordination centre requires detailed information about the controllable loads and time frames, in order to anticipate the effects of the according control signals. This approach seems to be more interesting for industrial consumers due to the complexity and heterogeneity of domestic household devices and appliances.

In the area of indirect load control very different approaches have been researched. Close to direct control is the use of price signals as described by [18]. It is based on the familiar top-down communication of price signals through a central control centre and therefore, a consequent enhancement of existing tariff models. But simple price minimization may cause peak-loads for automated consumers. Therefore, a real time observation is required so that the operator is able to counteract. Common are also auction-based approaches, where the consumer either bargains directly with the distributed energy resources or via aggregation agents [6], [16], [19]. All this approaches have in common, that the generator and load respectively the prices are tuned and negotiated in a bilateral way. A common challenge hereby is the a priori agreement on price barriers and acceptance levels for each DER or load through the producers and consumers. The last class of approaches focuses on the optimization on the consumer-side in order to generate an optimal degree of efficiency related to the according business- or operational-model. Both [20] and [21] describe exemplary use-cases with regard to specific business-models.

The usage of MAS for the aggregation of DERs to microgrids, modern, small-scale versions of the centralized electricity systems is described in [22], [23]. In [22] the aggregation of DERs and loads together to an autonomous entity a microgrid is described. Here a MAS approach as a branch of distributed artificial intelligence methods is introduced for DERs control in the microgrid. Similar work has been undone in [23] where a MAS for energy resource scheduling of an islanded power systems with DER is presented. It monitors, controls and operates an energy system consisting of a set of microgrids and lumped loads.

An interesting overview about the lastest applications of MAS in the smart grid context is given in [24].

\section{Distributed Multi-Agent Architecture}

Demand-Side applications usually contain large numbers of dynamic and inhomogeneous loads and thus require a distributed architecture with loose-coupled entities and generic models in order to handle the system-immanent complexities. Multi-Agents-Systems are a good metaphor for modeling and simulating such distributed grid components.

The abstract MAS architecture designed for such demand optimization problems shown in Figure 1 consists of four layers. The first layer is the market layer containing exchange markets for generation and energy services (see [4]). On a physical layer, each domestic household acts like an agent, monitoring the own environment through sensors, trying to achieve interests and goals (like a low energy price), while handling the system immanent restrictions (e.g. maintaining 


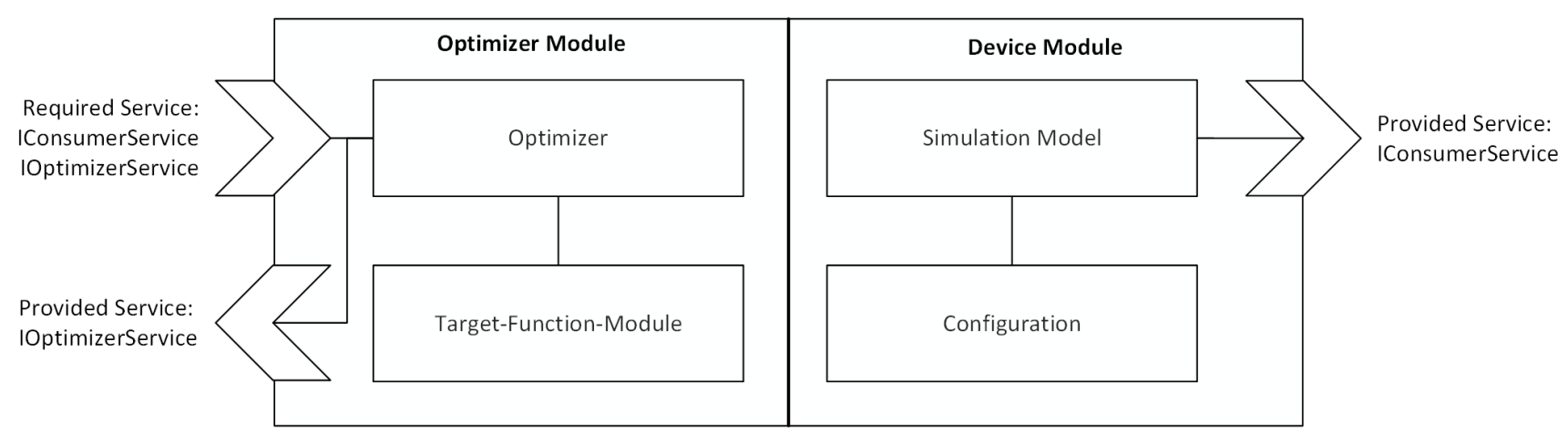

Fig. 2. Modular structure of the Device-Agent.

TABLE I

ClASSIFICATION OF LOADS

\begin{tabular}{|l|l|l|l|}
\hline Class & Description & Control & Examples \\
\hline $\begin{array}{l}\text { User-driven } \\
\text { loads }\end{array}$ & $\begin{array}{l}\text { Loads that satisfy the } \\
\text { users demand directly }\end{array}$ & $\begin{array}{l}\text { User- } \\
\text { controlled }\end{array}$ & Light, TV \\
\hline $\begin{array}{l}\text { Program- } \\
\text { driven } \\
\text { loads }\end{array}$ & $\begin{array}{l}\text { The user starts the de- } \\
\text { vice but it may not run } \\
\text { immediately }\end{array}$ & $\begin{array}{l}\text { semi- } \\
\text { automatic }\end{array}$ & $\begin{array}{l}\text { Washing } \\
\text { machines, } \\
\text { dishwasher }\end{array}$ \\
\hline $\begin{array}{l}\text { Fully- } \\
\text { automated } \\
\text { loads }\end{array}$ & $\begin{array}{l}\text { These devices have ac- } \\
\text { tors and sensors to } \\
\text { maintain a certain state }\end{array}$ & $\begin{array}{l}\text { Automatic, } \\
\text { parameter } \\
\text { driven }\end{array}$ & $\begin{array}{l}\text { Electric } \\
\text { heating or } \\
\text { cooling } \\
\text { (fridge) }\end{array}$ \\
\hline
\end{tabular}

a certain temperature in the household). This behavior, represented through Device-Agents, could be used by the Energy Management System as a virtual (and distributed) load to provide grid services to the market.

The Device-Agent (see Figure 2) is the most important entity in this energy system. Its role is to make the underlying physical layer transparent for the optimization system. With the Device-Agent as a gateway interface to the smart grid, it could abstract $1: n$ physical devices and appliances, providing the accumulated DSM-potential of the underlying physical layer (like load-shifting). The Device-Agent needs actors and sensors to control the physical devices as well as an intrinsic simulation model in order to anticipate the runtime constraints of the physical devices connected to it.

The load of the Device-Agent is described by:

$$
L\left\{\tau_{1}, \tau_{2}, \ldots, \tau_{n_{m}}\right\}=\sum_{i=0}^{n_{m}} \mathcal{L}\left(t-\tau_{i}\right),
$$

with $n_{m}$ as the variable number of starting times for the device, $\mathcal{L}\left(t-\tau_{i}\right)$ as the load of the agent, and $\tau_{i}$ as the discrete starting times of the loads.

An optional second module of the Device-Agent is the optimization module for the distributed, cooperative optimization of the loads. It implements a distributed lightweight optimization algorithm (described in Section IV) that optimizes the starting time of all participating Device-Agents utilizing the described iterative planning approach.

As stated in Table I loads could be classified into three groups [17]. The first class is only relevant for long term DSM efforts like strategic conservation [13]. The second and third classes can be planned iteratively using the provided IConsumerService of the related Device-Agent. An optimizer requests the first possible starting time vector from a DeviceAgent and receives it as an integer array, then chooses one starting time and commits it back to the Device-Agent. Depending on the chosen starting times the Device-Agent calculates the next possible runtime-vector with the simulation model. This will be repeated until the Device-Agent returns an end-of-planning-flag. Program-driven devices require just one planning step, as the first vector contains all possible starting times from the earliest to the latest. Under the assumption that every planning step of a fully-automated-device depends on the previous condition of it, the iterative planning provides a generic way to plan such devices. Thus, the optimizer is able to plan the device without domain-specific knowledge and the whole simulation and planning logic remains at the device for security reasons and separation of concern.

The Energy-Management-Agent (EMA) is the domain specific representation of the control component. It is responsible for planning and operation tasks and therefore, requires knowledge about the intended modus operandi and the current status of the associated Device-Agents. The role of the EMA in the Smart Grid could be a versatile one. For example, the EMA could aggregate Device-Agents all over the balancing zone for secondary or tertiary control. Focusing more on local business models, the EMA may optimize the load of domestic households towards the available power provided by DERs in a local grid.

The infrastructure domain is subdivided into two parts: The infrastructure represented by a simple TCP/IP-network supporting the service-based communication of the agents and a Registry-Agent. The latter one provides and manages information about the Device-Agents to the EMA as an additional ancillary service, so it is able to find suitable Device-Agents 
for the intended business model.

The Energy Market is a reactive agent or entity that provides a platform for an energy exchange. Here DERs can offer their generated power with the according prices, so consumers can evaluate and accept the offers.

At application runtime each Device-Agent has to register at the Registry-Agent giving information about its runtime capabilities, consumption capability, optional optimizer capability and some additional information (geographical or logical position in the grid). A User (Operator/Service Provider) defines the business plan parameters of the EMA i.e. the user group, markets or system targets. The EMA requests suitable Device-Agents at the Registry, reserves the control capability of the Device-Agents, defines a subset of optimizers out of the set of Device-Agents and parametrizes them (e.g. announces which Device-Agents are in the user group, which are the active/optimizing agents and which the passive ones). The optimizing Device-Agents iteratively plan the runtimes of all participating Device-Agents in the group and transmit the result to the EMA. The EMA then parametrizes every devices with its planed runtime.

\section{IMPLEMENTATION OF A DISTRIBUTED OPTIMIZATION ALGORITHM}

In order to optimize the starting times of the devices, every starting time vector submitted by a Device-Agent could be interpreted as a set of nodes in a directed graph (see Figure 3). Thus, each optimizing Device-Agent receives the same ordered list of Device-Agents to be optimized from the EMA, so they are all traversing the identical graph. The edges of this graph are weighted with the operational price.

This interpretation of the problem domain allows to apply several meta-heuristics for combinatorial optimization. Furthermore, to enable the consumer-side and to use the potential of the embedded-systems, the applied algorithms must be distributable, lightweight and scalable for different environmental conditions. Thus, Meta-heuristics like Evolutionary Algorithms (EA) with their population-based concepts seem to be suitable for such load planning problems.

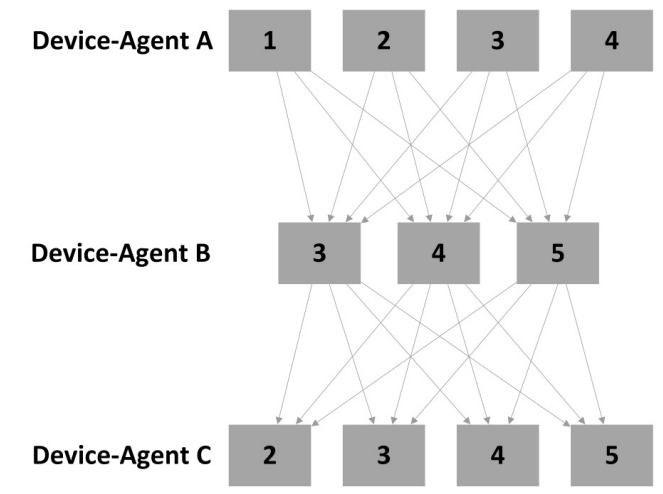

Fig. 3. Runtime vectors of three Device-Agents (A, B, C) interpreted as graph

\section{A. Definition of the optimization problem}

The optimization problem for load planning based on a power exchange market can be defined as the costminimization of the whole system:

$$
I=\min \sum_{t=0}^{T} \sum_{j=1}^{A} I_{j}(t) P_{j}(t),
$$

where $T$ is the number of time slots in the optimization period. Typically it ranges between 96 for quarter hour time slots and 1440 for minute-based time slots. $A$ denotes the number of offers for the prices $I_{j}(t)$, and $P_{j}(t)$ the amount of power bought from that offer. A constraint is: $P_{j}(t) \leq$ $P_{j, M A X}(t)$, meaning that the power bought may never exceed the offered amount.

The bought power is defined as:

$$
\sum_{j=1}^{A} P_{j}(t):=L_{N}\left\{\tau_{n}\right\}(t)
$$

with

$$
L_{N}\left\{\tau_{n}\right\}(t):=\sum_{m=0}^{M} L_{m}\left(\tau_{1}, \tau_{2}, \ldots, \tau_{n_{m}}\right)(t)
$$

where $M$ is the total number of devices and $L_{N}\left\{\tau_{n}\right\}(t)$ as the total consumption over all devices participating.

Propagating this problem to the virtual load by the EMS, the Device-Agents of the virtual load try to find cooperatively an optimal scheduling for price-minimization.

\section{B. The distributed ACS}

As a reference implementation an adapted version of the Ant Colony System (ACS, see [25]) was used. ACS is an algorithmic metaphor for food-searching ants, placing pheromones on their trail to indicate the shortest path between colony and food-source.

The problem described as a directed graph can be interpreted as a path-finding through the graph, a task ACS originally was designed for. In Figure 5 the global behavior of the optimizing module is illustrated. After the initialization phase, a Device-Agent (Master) with an optimizer module dispatches a certain number of Ant-Agents (Slaves) as its local colony. The local number of Ant-Agents and their serial or parallel execution (see [26]) depends on the properties and restrictions of each participating platform. Besides the nodes, the pheromones were also stored locally at the corresponding Device-Agent. Therefore, each Ant-Agent has to call the Device-Agent's service at least two times, one for the available nodes and one for the corresponding pheromone vector.

An Ant-Agent requests the initial vector of possible starting times from the first Device-Agent in the corresponding ordered list and randomly chooses the first staring time out of the vector and adds it to a temporary vector, before going iteratively through the following steps: 
1) It requests the next possible starting time vector and the pheromone vector from the Device-Agent, transmitting the previously chosen starting time.

2) If the vectors are available, the Ant Agent chooses the next node based on the costs of operation (edge-weight) and the according pheromones on the trail.

3) If the Agent receives an end-of-planning-Flag it proceeds to the next Device-Agent in the ordered list (if available) and executes step 1 .

Every optimizing Device-Agent has its own colony. After every full iteration, e.g. after each of the local Ant-Agent has traversed the graph once, the Master-Agent chooses the best-so-far path and places pheromones at the other DeviceAgents along the path through service calls. Because of the different computational speeds of the Device-Agents the pheromone adjustment happens asynchronously. Further synchronizing efforts are not required but fast optimizers may place pheromones more often than slower ones.

In a reference scenario with 100 domestic households, the ACS performs best compared to a basic Ant-System implementation [27] and a greedy algorithm. (see Figure 4).

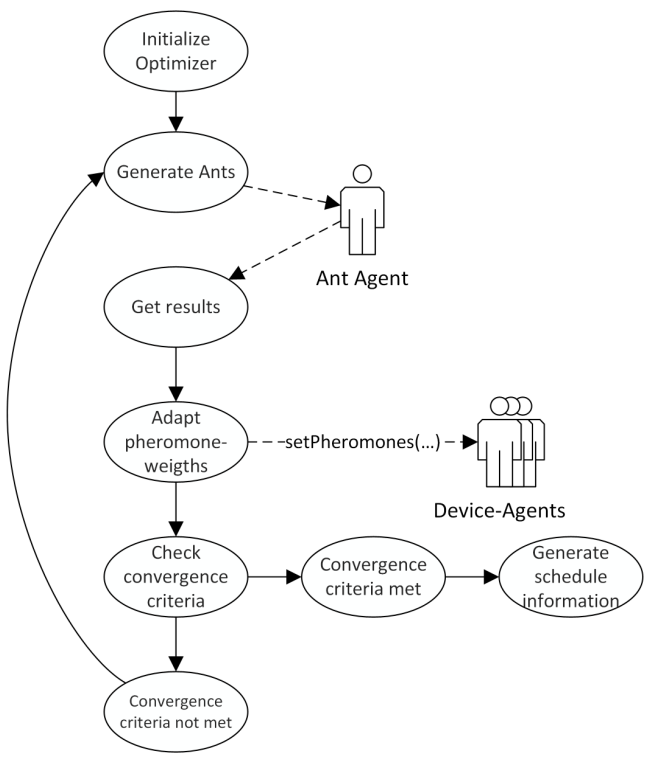

Fig. 5. Behavior of the device-agent optimizing the loads of other device agents.

\section{USE-CASE: PlanNing OF WIND ENERGY RESOURCES}

A typical use-case for consumer optimization is the dayahead optimization of predictable and controllable loads for a given load profile. It is often necessary to plan the power consumption according to the optimal utilization of volatile DERs to achieve a higher efficiency. Based on weather forecasts it is in many cases possible to predict the electricity generation capacity of wind turbines with a sufficient accuracy one day ahead. Energy generated by such plans is keen available, as the operation does not require any additional resources and thus the marginal costs are low. Therefore, if the demand is planned accordingly to the generated energy the users may save money through the efficient use of the resource as only a low amount of external (and often expensive) control energy has to be bought in addition.

For this use case example the generated energy of a 330 $\mathrm{kW}$ Enercon E-33 wind turbine is used to meet the demand of flexible consumers in a reference scenario. Although the DSM-potential of domestic households is often to be considered relatively low, the results can be easily transferred and compared, as industrial applications have often very specific requirements and constraints. The considered property consists of 100 active domestic households. Accordingly to the dispersion in Germany $92 \%$ of them have a washing machine, $62 \%$ a dish washer and $4 \%$ an electrical heater. This results in 158 program-driven and 4 full-automatic DeviceAgents representing and simulating the electrical consumers and appliances. The program-driven agents were statistically parameterized with regard to the Smart-A Project-Study [28]. The European-wide mean runtime probability of both washing machines and dish washers based on studies of the University of Bonn (Germany) were evaluated and implemented in the agent models. In order to calculate the runtime flexibility, the usage of power-up delays for the devices as described by the Smart-A Project-Study was analyzed. In case of the washing machines $56 \%$ of the users chose a power-up delay time period of 0 to 3 hours and $28 \%$ a time period of 4 to 6 hours. Therefore, by considering flexibilities up to six hours approx. $84 \%$ of the user preferences are considered in this use-case.

The study also shows the general acceptance of powerup delays for these type of devices. The broad degree of utilization alternates between approx. 38\% in Sweden up to $81 \%$ in Italy in which the actual usage of power-up delays for every stage of washing program goes to approx. $43 \%$. The situation for dish washers is similar. In $200739 \%$ of them were equipped with a power-up delay capability and $27 \%$ of the users were actually using it [28]. It can be assumed that the degree of usage and acceptance will increase further if the distribution of such power-up capabilities grows and more profitable DSM use-case utilizing this function will arise.

The fully-automated-device representing the electrical heater was parametrized with a temperature corridor of $1.0^{\circ} \mathrm{C}$ around the standard room temperature of $21^{\circ} \mathrm{C}$. The heater can be turned on and off again in 15 minute intervals. The outside temperature based on temperature data from the Hamburg's air quality control net for September 15th 2013.

The turbine's power generation was simulated with the Greenius-Tool ${ }^{5}$ based on weather data for the same day and scaled to the controllable power demand of 100 households in the reference property. So $10 \%$ of the turbine's nominal capacity can be used to meet the requirements of the flexible consumers. The wind power generation simulated here is extremely atypical for the property, where most of the energy is required in the evening hours due to the high amount of program-driven consumers. Therefore, even an optimized

${ }^{5}$ http://freegreenius.dlr.de 


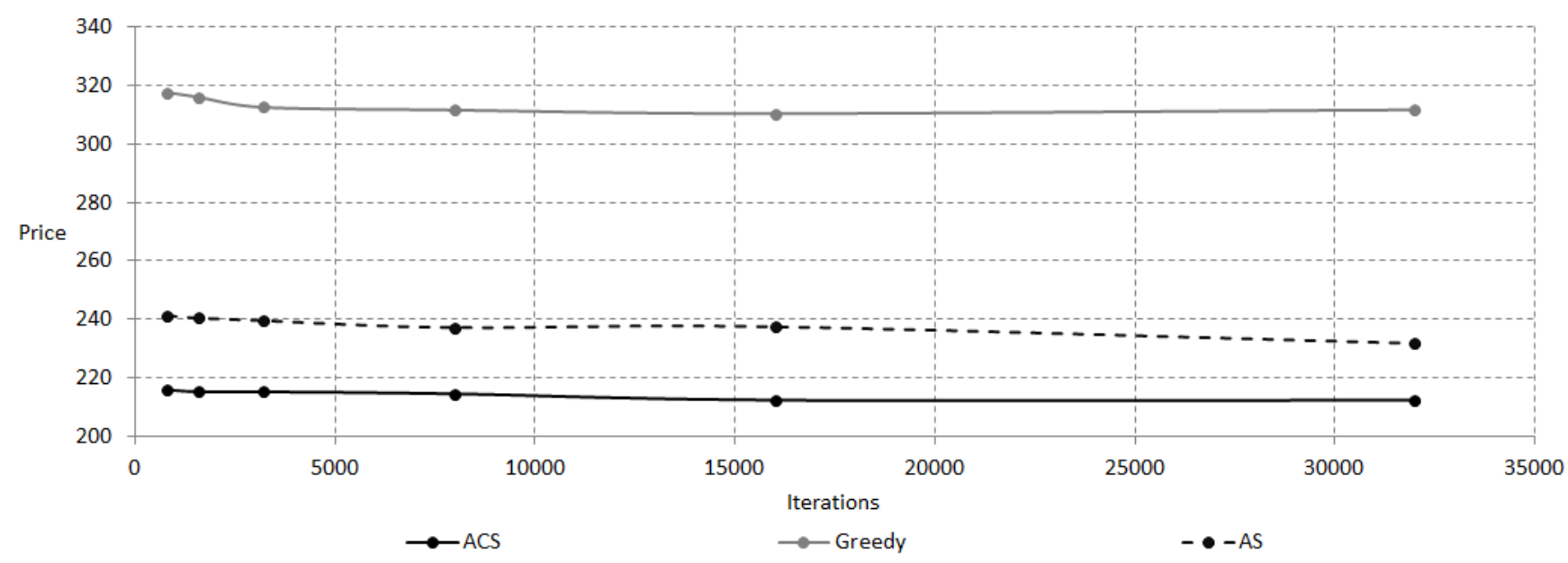

Fig. 4. Comparison between ACS, AS and a greedy algorithms.
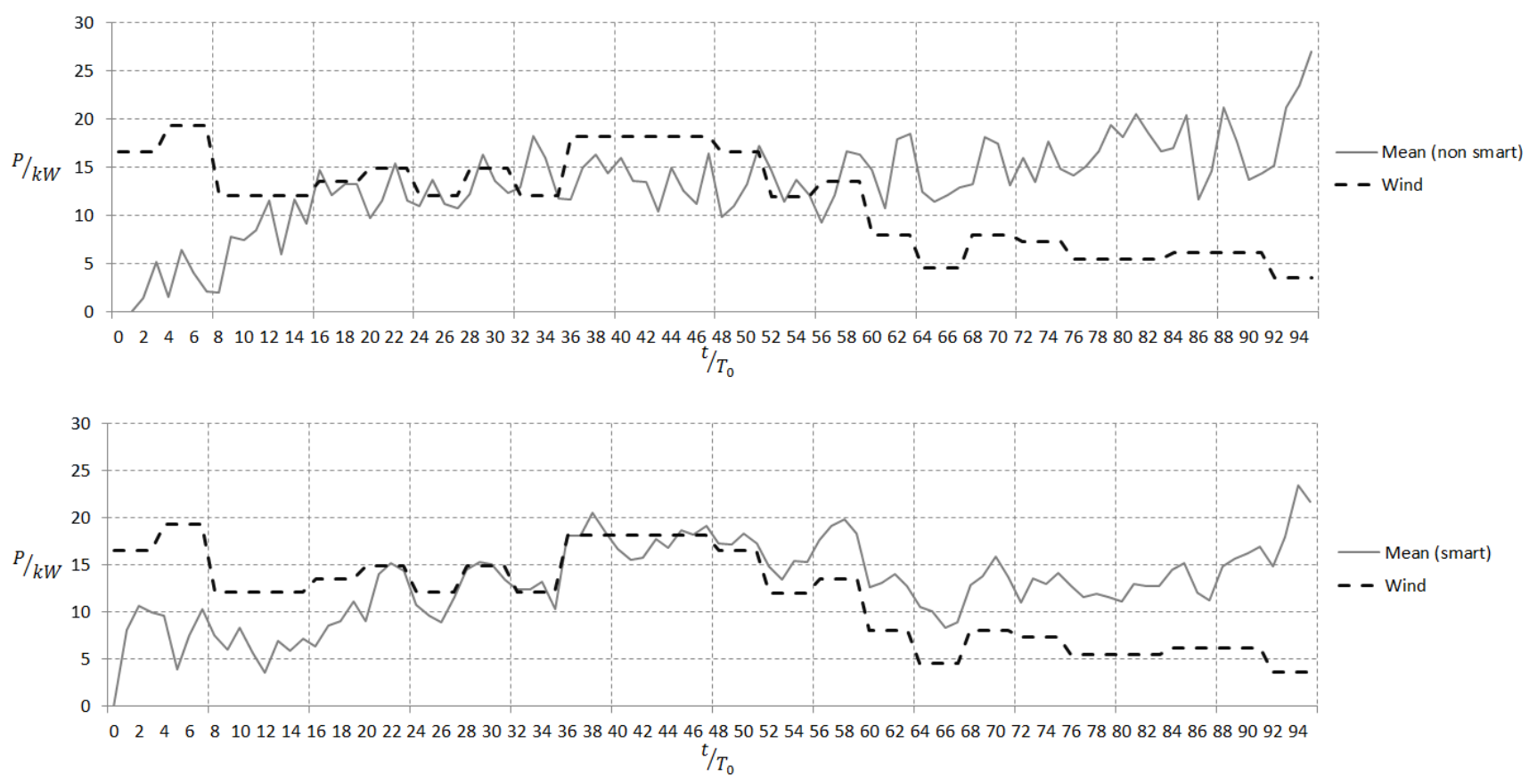

Fig. 6. Use-Case: Comparison of smart load control (below) and uncontrolled energy consumption (above).

schedule will not be able to utilize the available energy completely. But by utilizing the degrees of freedom of the four fully-automatic Device-Agents a significant energy saving can be achieved.

Figure 6 shows the aggregated results for 10 simulation runs. The upper chart shows the results for the uncontrolled energy consumption while the lower one displays the results of the ACS-based smart load optimization. For the latter parts of the day the energy consumption excels the generation as expected, but the optimized load control demands a significant lesser amount of external energy and utilizes especially earlier time slices better. The uncontrolled case utilizes $78,5 \%$ of the generated energy, while the controlled case uses $85,6 \%$ $(+7,1 \%)$. This implies that for the uncontrolled case 100,0 $\mathrm{kWh}$ of external energy have to been bought in addition, while the optimized scenario requires only $78,5 \mathrm{kWh}(-21,5$ $\mathrm{kWh}$ ). The addition of more fully-automated consumers, e.g. refrigerators or freezers should lead to further, significant improvements.

\section{CONCLUSiOn AND Future WORK}

In this paper a Demand-Side-Management oriented MultiAgent-System for distributed optimization was presented. The approach focusses on the automated use of flexibility potentials on the consumer-side. Through the use of a lightweight 
and distributed optimization concept the demand-side was integrated into the electricity market. The defined generic Device-Agent supports the planning of both program-driven and fully-automated devices without requiring domain specific knowledge about the optimizing agents. The exchange market model allows the application of many different use-cases, including e.g. flexible tariffs.

The implementation of Ant Colony System (ACS) as a metaheuristics for the optimization was described as well as the developed distribution concept of the algorithm. The presented use-case study includes 100 active domestic households with 158 Device-Agents and a wind turbine. The Device-Agents of the households optimized their demand towards the predicted power generation of the wind-turbine. It could be shown that the optimized planning lowered the demand for external energy about $21,5 \mathrm{kWh}$, even as the generation was extremely atypical for the normal load curve of the property.

Future work will cover several topics of the developed system. First, grid restrictions (avalanche-effect protection, see [20]) will be considered, which includes also the further development of the exchange market and its capabilities. A second aspect is to integrate a wider range of distributed optimization algorithms. Evolutionary Algorithms like Genetic Algorithms or other state of the art techniques of modern Operations Research like combined Branch-and-Cut and metaheuristics will be examined as first tests have shown that ACS finds very good solutions but leaves room for performance improvement. Also specification for regional market layers as well as generic energy-service descriptions of the typical use-cases could be defined. Furthermore, field tests and the integration of the proposed system as extension into systems like OGEMA or OpenADR can be targeted.

\section{REFERENCES}

[1] C. Mitchell, R. Deumling, and G. Court, "Stabilizing california's demand," Fortnightly Magazine, vol. 03, p. 10, 2009.

[2] U.S. Energy Information Administration, "Annual energy outlook 2013 with projections to 2040," 2013.

[3] Statistisches Bundesamt, "Wirtschaftsbereich energie - erzeugung," Statistisches Bundesamt, Tech. Rep., 2013.

[4] NIST, "Roadmap for smart grid interoperability standards," NIST special publication, vol. 1108, 2010.

[5] J. Bruinenberg, L. Colton, E. Darmois, J. Dorn, J. Doyle, O. Elloumi, H. Englert, R. Forbes, J. Heiles, P. Hermans et al., "Smart grid coordination group technical report reference architecture for the smart grid version 1.0 (draft) 2012-03-02," CEN, CENELEC, ETSI, Tech. Rep, 2012.

[6] J. Kok, C. Warmer, and I. Kamphuis, "Powermatcher: multiagent control in the electricity infrastructure," in Proceedings of the fourth international joint conference on Autonomous agents and multiagent systems. ACM, 2005. doi: http://dx.doi.org/10.1145/1082473.1082807 pp. 75-82.

[7] J. Bergmann, C. Glomb, J. Gotz, J. Heuer, R. Kuntschke, and M. Winter, "Scalability of smart grid protocols: Protocols and their simulative evaluation for massively distributed ders," in First IEEE International Conference on Smart Grid Communications (SmartGridComm). IEEE, 2010. doi: http://dx.doi.org/10.1109/SMARTGRID.2010.5622032 pp. 131 136.
[8] J. Kok, M. Scheepers, and I. Kamphuis, "Intelligence in electricity networks for embedding renewables and distributed generation," in Intelligent infrastructures. Springer, 2010, pp. 179-209.

[9] P. Palensky and D. Dietrich, "Demand side management: Demand response, intelligent energy systems, and smart loads," IEEE Transactions on Industrial Informatics, vol. 7, no. 3, pp. 381-388, 2011. doi: http://dx.doi.org/10.1109/TII.2011.2158841

[10] D. Nestle, J. Ringelstein, and H. Waldschmidt, "Open energy gateway architecture for customers in the distribution grid," Information Technology, Oldenbourg Verlag, Munich, pp. 83-88, 2010.

[11] M. A. Piette, G. Ghatikar, S. Kiliccote, P. Palensky, C. McParland, E. Koch, and D. Hennage, "Open automated demand response communications specification (version 1.0)."

[12] T. Dethlefs and W. Renz, "A distributed registry for service-based energy management systems," in 39th Annual Conference of the IEEE Industrial Electronics Society, IECON. IEEE, 2013. doi: http://dx.doi.org/10.1109/IECON.2013.6699896 pp. 4710-4714.

[13] C. W. Gellings, "The concept of demand-side management for electric utilities," Proceedings of the IEEE, vol. 73, no. 10, pp. 1468-1470, 1985. doi: http://dx.doi.org/0.1109/PROC.1985.13318

[14] Federal Energy Regulatory Commission, A National Assessment of Demand Response Potential, 2009.

[15] D. Huber, Z. Taylor, and S. Knudsen, "Environmental impacts of smart grid," National Energy Technology Laboratory, US-Department of Energy, Tech. Rep., 2011.

[16] T. Linnenberg, I. Wior, S. Schreiber, and A. Fay, "A market-based multiagent-system for decentralized power and grid control," in IEEE EFTA 2011, 2011. doi: http://dx.doi.org/0.1109/ETFA.2011.6059126

[17] O. Lünsdorf and M. Sonnenschein, "Lastadaption von haushaltsgeräten durch verbundsteuerung," in Tagungsband zum 3. Statusseminar des FEN. Forschungsverbund Energie Niedersachsen, 092009.

[18] S. Ramchurn, P. Vytelingum, and A. Rogers, "Agent-based control for decentralised demand side management in the smart grid," in The Tenth International Conference on Autonomous Agents and Multiagent Systems AAMAS 2011 (2011), 2011, pp. 5-12.

[19] S. Beer, M. Sonnenschein, and H.-J. Appelrath, "Towards a selforganization mechanism for agent associations in electricity spot markets," Informatik, 2011.

[20] A. Kamper, Dezentrales Lastmanagement zum Ausgleich kurzfristiger Abweichungen im Stromnetz. KIT Scientific Publishing, 2010.

[21] T. Logenthiran, D. Srinivasan, and T. Z. Shun, "Demand side management in smart grid using heuristic optimization," IEEE Transactions on Smart Grid, vol. 3, no. 3, pp. 1244-1252, 2012. doi: http://dx.doi.org/10.1109/TSG.2012.2195686

[22] W.-D. Zheng and J.-D. Cai, "A multi-agent system for distributed energy resources control in microgrid," in Critical Infrastructure (CRIS), 2010 5th International Conference on, Sept 2010. doi: http://dx.doi.org/10.1109/CRIS.2010.5617485 pp. 1-5.

[23] T. Logenthiran, D. Srinivasan, and A. M. Khambadkone, "Multi-agent system for energy resource scheduling of integrated microgrids in a distributed system," Electric Power Systems Research, vol. 81, no. 1, pp. 138 - 148, 2011. doi: http://dx.doi.org/10.1016/j.epsr.2010.07.019

[24] M. S. Narkhede, S. Chatterji, and S. Ghosh, "Article: Multi-agent systems (mas) controlled smart grid - a review," IJCA Proceedings on International Conference on Recent Trends in Engineering and Technology 2013, vol. ICRTET, no. 4, pp. 12-17, May 2013, published by Foundation of Computer Science, New York, USA.

[25] M. Dorigo and L. M. Gambardella, "Ant colony system: A cooperative learning approach to the traveling salesman problem," IEEE Transactions on Evolutionary Computation, vol. 1, no. 1, pp. 53-66, 1997.

[26] B. Bullnheimer, G. Kotsis, and C. Strauß, "Parallelization strategies for the ant system," 1997.

[27] M. Dorigo, V. Maniezzo, and A. Colorni, "Ant system: optimization by a colony of cooperation agents," IEEE Transactions on Systems Man and Cybernetics, vol. 26, pp. 29 - 41, 1996. doi: http://dx.doi.org/10.1109/3477.484436

[28] R. Stamminger, G. Broil, C. Pakula, H. Jungbecker, M. Braun, I. Rüdenauer, and C. Wendker, "Synergy potential of smart appliances," Report of the Smart-A project, 2008. 